\title{
以取代氨基硫腿为手臂的新型脱氧胆酸类分子钳的微波合成
}

\author{
夏振洋赵志刚* 石治川 刘 敏 \\ (西南民族大学化学与环境保护工程学院 成都 610041)
}

\begin{abstract}
摘要 在微波辐射条件下，以脱氧胆酸甲酯为隔离基，通过三光气桥连取代氨基硫艮为手臂，以良好的产率合成了 10 个新型脱氧胆酸分子钳，其结构均经 ${ }^{1} \mathrm{H} N \mathrm{NM}, \mathrm{IR}, \mathrm{MS}$ 及元素分析确证. 微波法具有反应时间短，产率高，对环境友好 等优点. 利用差紫外可见光光度滴定法测试了主体化合物对 $\mathrm{Cl}^{-}, \mathrm{Br}^{-}, \mathrm{I}^{-}, \mathrm{NO}_{3}^{-}$等阴离子的识别作用. 实验结果表明, 主体对阴离子有良好的识别性能.

关键词 脱氧胆酸; 分子钳; 微波合成; 分子识别
\end{abstract}

\section{Microwave-Assisted Synthesis of Novel Molecular Tweezers Based on Deoxycholic Acid with Arm of Substituted Thiosemi- carbazides}

\author{
Xia, Zhenyang Zhao, Zhigang* Shi, Zhichuan $\quad$ Liu, Min \\ (College of Chemistry and Environmental Protection Engineering, Southwest University for Nationalities, Chengdu 610041)
}

\begin{abstract}
Ten new molecular tweezers based on deoxycholic acid were synthesized in good yields by using deoxycholic acid methyl ester as spacer to bridge different substituted thiosemicarbazides via triphosgene under microwave irradiation. Their structures were charactetized by ${ }^{1} \mathrm{H}$ NMR, IR, MS techniques and elemental analysis. The microwave method has distinct advantages such as short reaction time, high yields and eco-friendly to the environment. The recognition properties of these molecular tweezers for $\mathrm{Cl}^{-}, \mathrm{Br}^{-}, \mathrm{I}^{-}, \mathrm{NO}_{3}^{-}$anions were investigated by $\mathrm{UV}$-vis spectra titration. The result indicated that this type of molecular tweezers has good binding properties for anions.
\end{abstract}

Keywords deoxycholic acid; molecular tweezer; microwave synthesis; molecular recognition

由于无机和有机阴离子在环境科学、生命科学、药 物化学以及催化化学等方面具有重要的作用, 因此在超 分子化学中对阴离子的识别研究引起了人们的极大兴 趣 ${ }^{[17]}$. 在众多的对阴离子识别的人工受体中, 钳形人 工受体具有结构灵活、易于修饰以及易于将裂穴内功能 团汇聚在受体与底物结合的活性部位上等优点而受到 人们的广泛关注 ${ }^{[8 \sim 10]}$.

胆甾由于其具有刚性的凹面结构和天然的不对称 性, 是构筑钳形人工受体的理想结构单元. 取代氨基硫 脲分子中有多个 $\mathrm{N}-\mathrm{H}$ 结构单元, 在分子识别过程中, 可与底物形成多重氢键. 迄今, 以取代氨基硫艮为手臂 的脱氧胆酸类分子钳在国内外罕见报道. 鉴于微波促进
的合成反应具有反应速度快、效率高、副产物少、对环 境友好等优点 ${ }^{[11 ~ 13]}$, 本工作在微波辐射条件下，以脱氧 胆酸甲酯为隔离基，取代氨基硫䐂为手臂，合成了一类 新型脱氧胆酸分子钳, 并考察了其对阴离子的识别性 能, 合成路线见 Scheme 1.

\section{1 实验部分}

\section{1 仪器与试剂}

核磁共振仪: Varian INOVA-400 MHz, $\mathrm{Me}_{4} \mathrm{Si}$ 作内 标, $\mathrm{CDCl}_{3}$ 为溶剂; 质谱仪: FINNIGAN-LCQ ${ }^{\mathrm{DECA}}$ 型; 红 外光谱仪: PERKINEL-MER1700 型, KBr 压片; 紫外可 见光谱仪为北京普析 TU-1901 型分光光度计; 元素分析

\footnotetext{
*E-mail: zzg63129@yahoo.com.cn

Received August 27, 2012; revised September 27, 2012; published online October 9, 2012.
}

Project supported by the Fundamental Research Funds of Central Universities, Southwest University for Nationalities (Nos. 12ZYXS77, 11NZYTH05), the Science and Technology Department of Sichuan Province (No. 2012SZ0160) and the National Foreign Expert Bureau (No. 2012-29).

西南民族大学中央高校基本科研业务费科学基金(Nos. 12ZYXS77, 11NZYTH05)、四川省科技支撑计划基金(No. 2012SZ0160)和国家外专局基金(No. 2012-29)资助项目. 


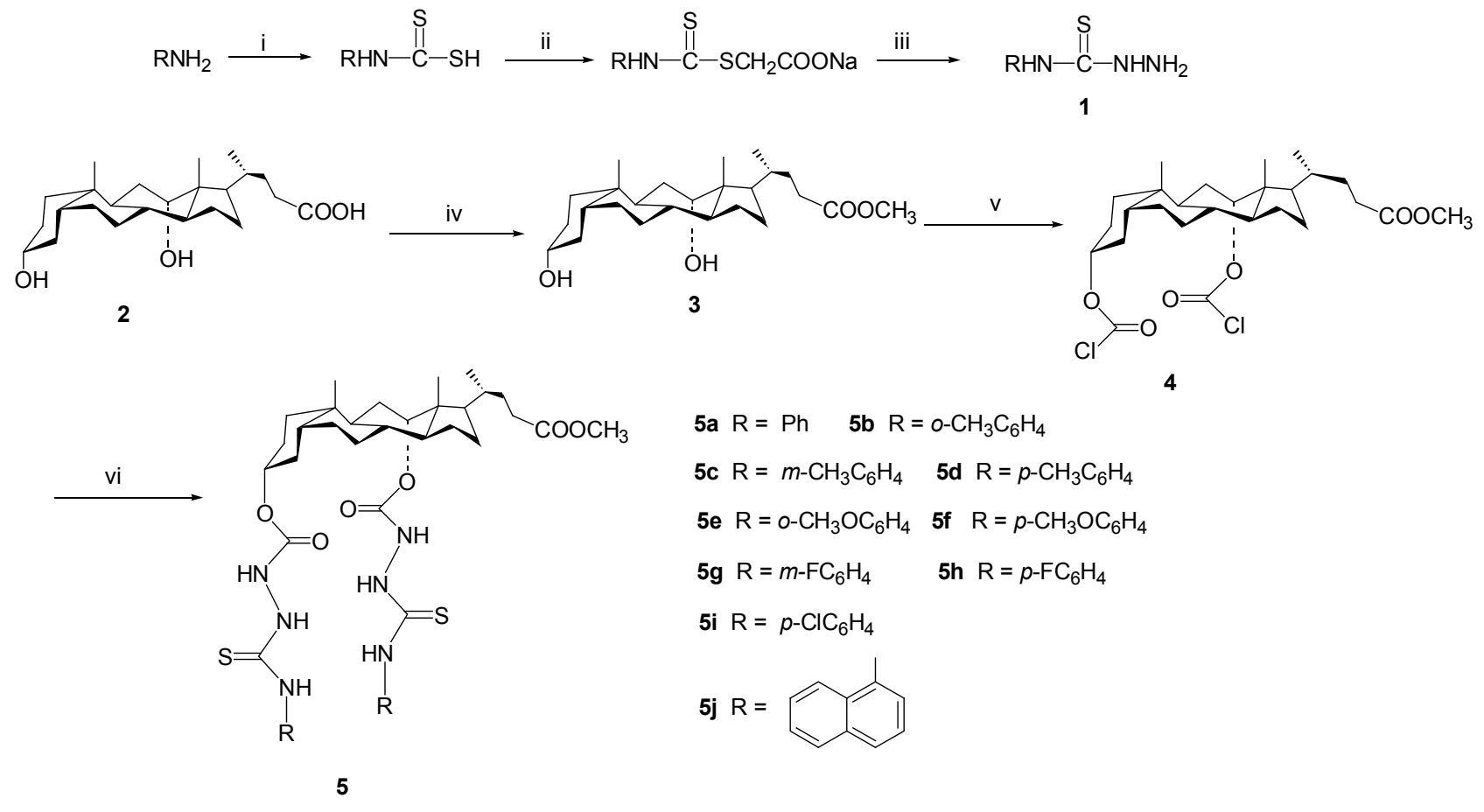

Reagents and conditions: (i) $\mathrm{CS}_{2}, \mathrm{NH}_{3} \cdot \mathrm{H}_{2} \mathrm{O}$; (ii) $\mathrm{ClCH}_{2} \mathrm{COONa}$; (iii) $\mathrm{N}_{2} \mathrm{H}_{4} \cdot \mathrm{H}_{2} \mathrm{O}$; (iv) $\mathrm{CH}_{3} \mathrm{OH}, \mathrm{CH}_{3} \mathrm{COCl}$; (v) triphosgene, $\mathrm{CH}_{2} \mathrm{Cl}_{2}$, pyridine, $\mathrm{MW}$; (vi) pyridine, intermediate $1, \mathrm{MW}$

\section{Scheme 1}

仪: Carlo-Erba-1106 型; 旋光仪: WZZ-2B 自动旋光仪; 熔点仪: X-4 型数字显示显微熔点测定仪; 微波反应器: 北京祥鹄科技公司商用微波反应器 XH-100A.

所用试剂均为市售化学纯或分析纯. 硅胶 $\mathrm{H}$ 、硅胶 $\mathrm{GF}_{254}$ : 青岛海洋化工厂生产; 无水 $\mathrm{CH}_{2} \mathrm{Cl}_{2}$ : 加 $\mathrm{CaH}_{2}$ 回 流数天后, 反应前新蒸使用; 无水吡啶: 加 $\mathrm{KOH}$ 干燥 $24 \mathrm{~h}$ 后, 蒸馏收集 $114 \sim 116{ }^{\circ} \mathrm{C}$ 馏分; 三光气: 工业用 品; $(n-\mathrm{Bu})_{4} \mathrm{~N}^{+} \mathrm{I}^{-}$试剂为上海试剂一厂生产, $(n-\mathrm{Bu})_{4} \mathrm{~N}^{+} \mathrm{Cl}^{-}$ 为上海盈元化工有限公司生产, $(n-\mathrm{Bu})_{4} \mathrm{~N}^{+} \mathrm{Br}^{-}$为成都科 龙化工试剂厂生产, $(n-\mathrm{Bu})_{4} \mathrm{~N}^{+} \mathrm{NO}_{3}^{-}$为 Alfa Aesar 生产, 中间体 1 和 3 按文献 $[14,15]$ 的方法合成.

\section{2 分子钳 $5 a \sim 5 j$ 的微波合成与表征}

在 $50 \mathrm{~mL}$ 三颈瓶中加入 $0.2 \mathrm{~g}(0.5 \mathrm{mmol})$ 脱氧胆酸甲 酯中间体 3,10 mL 无水 $\mathrm{CH}_{2} \mathrm{Cl}_{2}, 0.2 \mathrm{~mL}$ 无水吡啶, $0.11 \mathrm{~g}$ (0.37 mmol)三光气, 将三颈瓶放入微波炉中, 装上配有 干燥管的冷凝管, 于 $150 \mathrm{~W}$ 下回流反应 $10 \mathrm{~min}$, 待反应 物冷却至室温, 相继加入 $1.2 \mathrm{mmol}$ 中间体 $\mathbf{1}$ 和 $0.2 \mathrm{~mL}$ 无水吡啶，继续在相同功率下微波辐射回流反应 10 $15 \mathrm{~min}$ (TLC 监测反应进程). 反应完成后, 减压蒸去 $\mathrm{CH}_{2} \mathrm{Cl}_{2}$, 残余物加入 $20 \mathrm{~mL}$ 乙酸乙酯溶解, 过滤, 滤渣 用 $10 \mathrm{~mL}$ 乙酸乙酯洗涤, 合并滤液, 依次用 $5 \% \mathrm{NaHCO}_{3}$ $(15 \mathrm{~mL} \times 3)$, 饱和食盐水 $(15 \mathrm{~mL} \times 3)$ 洗涤, 无水 $\mathrm{Na}_{2} \mathrm{SO}_{4}$ 干燥, 过滤, 减压蒸去溶剂, 柱层析分离纯化[固定相用 硅胶 $\mathrm{H}$, 洗脱剂为 $V$ (石油醚) $: V\left(\mathrm{CH}_{3} \mathrm{COOC}_{2} \mathrm{H}_{5}\right)=8$ :
$1 \sim 1 ： 1$ ] 得纯品.

$3 \alpha, 12 \alpha$-二 $\left[\left(N\right.\right.$ - 苯基- $N^{\prime}$-氨基甲酰氧基 $\left.)\right]$ 硫脲-5 $\beta-24-$ 胆烷酸甲酯(5a): 蓝色晶体, 收率 83\%. m.p. 75 76 ${ }^{\circ} \mathrm{C}$; $[\alpha]_{\mathrm{D}}^{20}-26.32\left(c \quad 0.12, \mathrm{CH}_{2} \mathrm{Cl}_{2}\right) ;{ }^{1} \mathrm{H} \mathrm{NMR}\left(\mathrm{CDCl}_{3}, 400\right.$ $\mathrm{MHz}) \delta: 10.51(\mathrm{~s}, 1 \mathrm{H}, \mathrm{NH}), 10.12(\mathrm{~s}, 1 \mathrm{H}, \mathrm{NH}), 8.44$ (s, 1H, $\mathrm{NH}), 8.26$ (s, 1H, NH), 8.01 (s, 1H, NH), 7.79 (s, 1H, NH), $7.52 \sim 7.20(\mathrm{~m}, 10 \mathrm{H}$, ArH), 5.03 (brs, $1 \mathrm{H}, 12 \beta-\mathrm{H}), 4.63$ (brs, $1 \mathrm{H}, 3 \beta-\mathrm{H}), 3.60\left(\mathrm{~s}, 3 \mathrm{H}, \mathrm{COOCH}_{3}\right), 0.94(\mathrm{~s}, 3 \mathrm{H}$, $\left.19-\mathrm{CH}_{3}\right), 0.80\left(\mathrm{~d}, J=6.4 \mathrm{~Hz}, 3 \mathrm{H}, 21-\mathrm{CH}_{3}\right), 0.72(\mathrm{~s}, 3 \mathrm{H}$, $18-\mathrm{CH}_{3}$ ); IR (KBr) v: $3288,2926,2867,1723,1596,1545$, $1256 \mathrm{~cm}^{-1}$; ESI-MS m/z (\%): $793\left[(\mathrm{M}+\mathrm{H})^{+}, 100\right]$. Anal. calcd for $\mathrm{C}_{41} \mathrm{H}_{56} \mathrm{~N}_{6} \mathrm{O}_{6} \mathrm{~S}_{2}$ : C 62.09, H 7.12, N 10.60; found C 62.21, H 7.13, N 10.62.

$3 \alpha, 12 \alpha$-二 $\left[\left(N\right.\right.$-邻甲基苯基- $N^{\prime}$-氨基甲酰氧基 $\left.)\right]$ 硫脲5 $\beta$-24-胆烷酸甲酯 $(\mathbf{5 b})$ : 黄色晶体，收率 82\%. m.p. 96 $97{ }^{\circ} \mathrm{C} ;[\alpha]_{\mathrm{D}}^{20}+36.36\left(c 0.10, \mathrm{CH}_{2} \mathrm{Cl}_{2}\right) ;{ }^{1} \mathrm{H} \mathrm{NMR}\left(\mathrm{CDCl}_{3}\right.$, $400 \mathrm{MHz}) \delta: 10.47$ (s, 1H, NH), 10.01 (s, 1H, NH), 8.19 (s, 1H, NH), 7.84 (s, 1H, NH), 7.66 (s, 1H, NH), 7.45 (s, 1H, NH), $7.26 \sim 7.11(\mathrm{~m}, 8 \mathrm{H}, \mathrm{ArH}), 4.97$ (brs, $1 \mathrm{H}, 12 \beta-\mathrm{H})$, 4.68 (brs, $1 \mathrm{H}, 3 \beta-\mathrm{H}), 3.66$ (s, 3H, $\left.\mathrm{COOCH}_{3}\right), 2.33(\mathrm{~s}, 3 \mathrm{H}$, $\left.\mathrm{ArCH}_{3}\right), 2.26$ (s, 3H, $\left.\mathrm{ArCH}_{3}\right), 0.95$ (s, 3H, 19- $\left.\mathrm{CH}_{3}\right), 0.79$ (d, $\left.J=6.4 \mathrm{~Hz}, 3 \mathrm{H}, 21-\mathrm{CH}_{3}\right), 0.71\left(\mathrm{~s}, 3 \mathrm{H}, 18-\mathrm{CH}_{3}\right)$; IR (KBr) v: 3359, 2930, 2865, 1728 1527, 1456, $1221 \mathrm{~cm}^{-1}$; ESI-MS $m / z(\%): 843\left[(\mathrm{M}+\mathrm{Na})^{+}, 100\right]$. Anal. calcd for $\mathrm{C}_{43} \mathrm{H}_{60} \mathrm{~N}_{6} \mathrm{O}_{6} \mathrm{~S}_{2}$ : C 62.90, H 7.37, N 10.24; found C 62.73, H 
7.39, N 10.22.

$3 \alpha, 12 \alpha$-二 $\left[\left(N\right.\right.$-间甲基苯基- $N^{\prime}$-氨基甲酰氧基 $\left.)\right]$ 硫脲5 $\beta$-24-胆烷酸甲酯 $(\mathbf{5 c})$ : 绿色晶体, 收率 86\%. m.p. 111 $112{ }^{\circ} \mathrm{C} ;[\alpha]_{\mathrm{D}}^{20}+28.17\left(c 0.11, \mathrm{CH}_{2} \mathrm{Cl}_{2}\right) ;{ }^{1} \mathrm{H} \mathrm{NMR}\left(\mathrm{CDCl}_{3}\right.$, $400 \mathrm{MHz}) \delta: 10.39$ (s, 1H, NH), 10.00 (s, 1H, NH), 8.20 (s, $1 \mathrm{H}, \mathrm{NH}), 7.83$ (s, 1H, NH), 7.64 (s, 1H, NH), 7.46 (s, 1H, $\mathrm{NH}), 7.16 \sim 6.89(\mathrm{~m}, 8 \mathrm{H}, \mathrm{ArH}), 5.04$ (brs, $1 \mathrm{H}, 12 \beta-\mathrm{H})$, 4.65 (brs, 1H, 3 $\beta-\mathrm{H}), 3.61$ (s, 3H, $\left.\mathrm{COOCH}_{3}\right), 2.31$ (s, 3H, $\left.\mathrm{ArCH}_{3}\right), 2.25\left(\mathrm{~s}, 3 \mathrm{H}, \mathrm{ArCH}_{3}\right), 0.92\left(\mathrm{~s}, 3 \mathrm{H}, 19-\mathrm{CH}_{3}\right), 0.77$ (d, $\left.J=6.4 \mathrm{~Hz}, 3 \mathrm{H}, 21-\mathrm{CH}_{3}\right), 0.70$ (s, 3H, H-18); IR (KBr) v: 3363, 2928, 1724, 1596, 1547, $1231 \mathrm{~cm}^{-1}$; ESI-MS m/z (\%): $821\left[(\mathrm{M}+\mathrm{H})^{+}, 100\right]$. Anal. calcd for $\mathrm{C}_{43} \mathrm{H}_{60} \mathrm{~N}_{6} \mathrm{O}_{6} \mathrm{~S}_{2}: \mathrm{C}$ 62.90, H 7.37, N 10.24; found C 62.99, H 7.35, N 10.26.

$3 \alpha, 12 \alpha$-二 $[(N$-对甲基苯基- $N$-氨基甲酰氧基 $)]$ 硫脲$5 \beta$-24-胆烷酸甲酯 $(\mathbf{5 d})$ : 橙色晶体, 收率 $85 \%$. m.p. $122 \sim 123{ }^{\circ} \mathrm{C} ;[\alpha]_{\mathrm{D}}^{20}+26.14\left(c \quad 0.12, \mathrm{CH}_{2} \mathrm{Cl}_{2}\right) ;{ }^{1} \mathrm{H}$ NMR $\left(\mathrm{CDCl}_{3}, 400 \mathrm{MHz}\right) \delta: 10.46(\mathrm{~s}, 1 \mathrm{H}, \mathrm{NH}), 10.02(\mathrm{~s}, 1 \mathrm{H}$, $\mathrm{NH}), 8.21$ (s, 1H, NH), 7.84 (s, 1H, NH), 7.66 (s, 1H, NH), 7. 45 (s, 1H, NH), $7.32 \sim 7.10(\mathrm{~m}, 8 \mathrm{H}, \mathrm{ArH}), 5.01$ (brs, $1 \mathrm{H}$, $12 \beta-\mathrm{H}), 4.60$ (brs, $1 \mathrm{H}, 3 \beta-\mathrm{H}), 3.61$ (s, 3H, $\left.\mathrm{COOCH}_{3}\right), 2.35$ $\left(\mathrm{s}, 3 \mathrm{H}, \mathrm{ArCH}_{3}\right), 2.27\left(\mathrm{~s}, 3 \mathrm{H}, \mathrm{ArCH}_{3}\right), 0.90\left(\mathrm{~s}, 3 \mathrm{H}, 19-\mathrm{CH}_{3}\right)$, 0.79 (d, $J=6.4 \mathrm{~Hz}, 3 \mathrm{H}, 21-\mathrm{CH}_{3}$ ), 0.69 (s, 3H, H-18); IR (KBr) v: 3290, 2939, 2867, 1728, 1597, 1526, $1217 \mathrm{~cm}^{-1}$; ESI-MS $m / z(\%): 821\left[(\mathrm{M}+\mathrm{H})^{+}, 100\right]$. Anal. calcd for $\mathrm{C}_{43} \mathrm{H}_{60} \mathrm{~N}_{6} \mathrm{O}_{6} \mathrm{~S}_{2}$ : C 62.90, H 7.37, N 10.24; found C 62.76, H 7.39, N 10.21 .

$3 \alpha, 12 \alpha$-二[( $N$-邻甲氧基苯基- $N^{\prime}$-氨基甲酰氧基 $\left.)\right]$ 硫 嫝-5 $\beta$-24-胆烷酸甲酯 $(5 \mathrm{e})$ : 黄色晶体, 收率 $82 \%$. m.p. $110 \sim 111{ }^{\circ} \mathrm{C} ;[\alpha]_{\mathrm{D}}^{20}-41.67\left(c \quad 0.14, \mathrm{CH}_{2} \mathrm{Cl}_{2}\right) ;{ }^{1} \mathrm{H}$ NMR $\left(\mathrm{CDCl}_{3}, 400 \mathrm{MHz}\right) \delta: 10.23$ (s, 1H, NH), 9.98 (s, 1H, NH), 8.25 (s, 1H, NH), 7.87 (s, 1H, NH), 7.63 (s, 1H, NH), 7.47 $(\mathrm{s}, 1 \mathrm{H}, \mathrm{NH}), 7.65 \sim 6.98(\mathrm{~m}, 8 \mathrm{H}, \mathrm{ArH}), 5.05$ (brs, $1 \mathrm{H}$, $12 \beta-\mathrm{H}), 4.64$ (brs, $1 \mathrm{H}, 3 \beta-\mathrm{H}), 3.89$ (s, 3H, $\left.\mathrm{ArOCH}_{3}\right), 3.86$ $\left(\mathrm{s}, 3 \mathrm{H}, \mathrm{ArOCH}_{3}\right), 3.63\left(\mathrm{~s}, 3 \mathrm{H}, \mathrm{COOCH}_{3}\right), 0.89(\mathrm{~s}, 3 \mathrm{H}$, $\left.19-\mathrm{CH}_{3}\right), 0.77$ (d, $\left.J=6.4 \mathrm{~Hz}, 3 \mathrm{H}, 21-\mathrm{CH}_{3}\right), 0.68(\mathrm{~s}, 3 \mathrm{H}$, H-18); IR (KBr) v: 3438, 2963, 2867, 1731, 1544, 1461 $1241 \mathrm{~cm}^{-1}$; ESI-MS m/z (\%): $853\left[(\mathrm{M}+\mathrm{H})^{+}, 100\right]$. Anal. calcd for $\mathrm{C}_{43} \mathrm{H}_{60} \mathrm{~N}_{6} \mathrm{O}_{8} \mathrm{~S}_{2}$ : C 60.54, H 7.09, N 9.85; found $\mathrm{C}$ $60.40, \mathrm{H} 7.11$, N 9.88 .

$3 \alpha, 12 \alpha$-二 $\left[\left(N\right.\right.$-对甲氧基苯基- $N^{\prime}$-氨基甲酰氧基 $\left.)\right]$ 硫 嫝-5 $\beta$-24-胆烷酸甲酯 $(\mathbf{5 f})$ : 绿色晶体，收率 $84 \%$. m.p. $135 \sim 136{ }^{\circ} \mathrm{C} ;[\alpha]_{\mathrm{D}}^{20}-29.43\left(c \quad 0.12, \mathrm{CH}_{2} \mathrm{Cl}_{2}\right) ;{ }^{1} \mathrm{H}$ NMR $\left(\mathrm{CDCl}_{3}, 400 \mathrm{MHz}\right) \delta: 10.45(\mathrm{~s}, 1 \mathrm{H}, \mathrm{NH}), 10.02(\mathrm{~s}, 1 \mathrm{H}$, $\mathrm{NH}), 8.27$ (s, 1H, NH), 7.89 (s, 1H, NH), 7.65 (s, 1H, NH), 7.49 (s, 1H, NH), $7.56 \sim 6.99$ (m, 8H, ArH), 5.02 (brs, 1H,

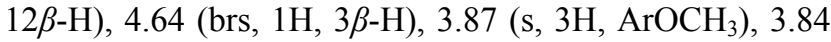
$\left(\mathrm{s}, 3 \mathrm{H}, \mathrm{ArOCH}_{3}\right), 3.64\left(\mathrm{~s}, 3 \mathrm{H}, \mathrm{COOCH}_{3}\right), 0.91(\mathrm{~s}, 3 \mathrm{H}$, $\left.19-\mathrm{CH}_{3}\right), 0.78$ (d, $\left.J=6.4 \mathrm{~Hz}, 3 \mathrm{H}, 21-\mathrm{CH}_{3}\right), 0.69$ (s, 3H, H-18); IR (KBr) v: 3362, 2936, 2867, 1727, 1517, 1458 $1233 \mathrm{~cm}^{-1}$; ESI-MS m/z (\%): $851\left[(\mathrm{M}-\mathrm{H})^{+}, 100\right]$. Anal. calcd for $\mathrm{C}_{43} \mathrm{H}_{60} \mathrm{~N}_{6} \mathrm{O}_{8} \mathrm{~S}_{2}$ : C 60.54, H 7.09, N 9.85; found C 60.63, H 7.08, N 9.82.

$3 \alpha, 12 \alpha$-二 $\left[\left(N\right.\right.$ - 间氟苯基- $N^{\prime}$-氨基甲酰氧基 $\left.)\right]$ 硫嫝5 $\beta$-24-胆烷酸甲酯 $(\mathbf{5 g})$ ：黄色晶体，收率 81\%. m.p. 97 $98{ }^{\circ} \mathrm{C} ;[\alpha]_{\mathrm{D}}^{20}+65.22\left(c 0.13, \mathrm{CH}_{2} \mathrm{Cl}_{2}\right) ;{ }^{1} \mathrm{H} \mathrm{NMR}\left(\mathrm{CDCl}_{3}\right.$, $400 \mathrm{MHz}) \delta: 10.55$ (s, 1H, NH), 10.24 (s, 1H, NH), 8.40 (s, 1H, NH), 8.05 (s, 1H, NH), 7.73 (s,1 H, NH), 7.55 (s, 1H, $\mathrm{NH}), 7.31 \sim 6.96(\mathrm{~m}, 8 \mathrm{H}, \mathrm{ArH}), 5.07$ (brs, $1 \mathrm{H}, 12 \beta-\mathrm{H})$, 4.65 (brs, $1 \mathrm{H}, 3 \beta-\mathrm{H}), 3.61$ (s, $\left.3 \mathrm{H}, \mathrm{COOCH}_{3}\right), 0.95(\mathrm{~s}, 3 \mathrm{H}$, $\left.19-\mathrm{CH}_{3}\right), 0.82\left(\mathrm{~d}, J=6.4 \mathrm{~Hz}, 3 \mathrm{H}, 21-\mathrm{CH}_{3}\right), 0.73$ (s, 3H, $\left.18-\mathrm{CH}_{3}\right)$; IR (KBr) v: 3273, 2943, 2869, 1723, 1608, 1541, $1233 \mathrm{~cm}^{-1}$; ESI-MS m/z (\%): $829\left[(\mathrm{M}+\mathrm{H})^{+}, 100\right]$. Anal. calcd for $\mathrm{C}_{41} \mathrm{H}_{54} \mathrm{~F}_{2} \mathrm{~N}_{6} \mathrm{O}_{6} \mathrm{~S}_{2}$ : C 59.40, $\mathrm{H}$ 6.57, $\mathrm{N}$ 10.14; found $\mathrm{C} 59.51, \mathrm{H} 6.58, \mathrm{~N} 10.11$.

$3 \alpha, 12 \alpha$-二 $\left[\left(N\right.\right.$ - 对氟苯基- $N^{\prime}$-氨基甲酰氧基 $\left.)\right]$ 硫嫝5 $\beta$-24-胆烷酸甲酯 $(5 \mathrm{~h})$ : 黄色晶体, 收率 $83 \%$. m.p. $124 \sim 125{ }^{\circ} \mathrm{C} ;[\alpha]_{\mathrm{D}}^{20}-12.85\left(c 0.15, \mathrm{CH}_{2} \mathrm{Cl}_{2}\right) ;{ }^{1} \mathrm{H}$ NMR $\left(\mathrm{CDCl}_{3}, 400 \mathrm{MHz}\right) \delta: 10.40(\mathrm{~s}, 1 \mathrm{H}, \mathrm{NH}), 10.09$ (s, $1 \mathrm{H}$, $\mathrm{NH}), 8.45$ (s, 1H, NH), 8.01 (s, 1H, NH), 7.80 (s, 1H, NH), 7.61 (s, 1H, NH), $7.39 \sim 6.99(\mathrm{~m}, 4 \mathrm{H}, \mathrm{ArH}), 5.04$ (brs, $1 \mathrm{H}$, $12 \beta-\mathrm{H}), 4.59$ (brs, $1 \mathrm{H}, 3 \beta-\mathrm{H}), 3.60$ (s, 3H, $\left.\mathrm{COOCH}_{3}\right), 0.93$ $\left(\mathrm{s}, 3 \mathrm{H}, 19-\mathrm{CH}_{3}\right), 0.81\left(\mathrm{~d}, J=6.4 \mathrm{~Hz}, 3 \mathrm{H}, 21-\mathrm{CH}_{3}\right), 0.70$ (s, $\left.3 \mathrm{H}, 18-\mathrm{CH}_{3}\right)$; IR (KBr) v: 3279, 2939, 2867, 1726, 1512, 1444, $1219 \mathrm{~cm}^{-1}$; ESI-MS m/z (\%): $829\left[(\mathrm{M}+\mathrm{H})^{+}, 100\right]$. Anal. calcd for $\mathrm{C}_{41} \mathrm{H}_{54} \mathrm{~F}_{2} \mathrm{~N}_{6} \mathrm{O}_{6} \mathrm{~S}_{2}$ : C 59.40, H 6.57, N 10.14; found $\mathrm{C} 59.49$, $\mathrm{H} 6.55, \mathrm{~N} 10.16$.

$3 \alpha, 12 \alpha-$ 二 $\left[\left(N-\right.\right.$ 对氯苯基- $N^{\prime}$-氨基甲酰氧基 $\left.)\right]$ 硫脲$5 \beta$-24-胆烷酸甲酯 $\mathbf{5 i}$ i): 棕黄色晶体，收率 $84 \%$. m.p. $70 \sim 71{ }^{\circ} \mathrm{C} ;[\alpha]_{\mathrm{D}}^{20}+35.01\left(c \quad 0.14, \mathrm{CH}_{2} \mathrm{Cl}_{2}\right) ;{ }^{1} \mathrm{H}$ NMR $\left(\mathrm{CDCl}_{3}, 400 \mathrm{MHz}\right) \delta: 10.54(\mathrm{~s}, 1 \mathrm{H}, \mathrm{NH}), 10.22(\mathrm{~s}, 1 \mathrm{H}$, $\mathrm{NH}), 8.42$ (s, 1H, NH), 8.02 (s, 1H, NH), 7.74 (s, 1H, NH), $7.51(\mathrm{~s}, 1 \mathrm{H}, \mathrm{NH}), 7.46 \sim 7.21(\mathrm{~m}, 8 \mathrm{H}, \mathrm{ArH}), 5.00$ (brs, $1 \mathrm{H}$, $12 \beta-\mathrm{H}), 4.63$ (brs, $1 \mathrm{H}, 12 \beta-\mathrm{H}), 3.63$ (s, $\left.3 \mathrm{H}, \mathrm{COOCH}_{3}\right)$, $0.96\left(\mathrm{~s}, 3 \mathrm{H}, 19-\mathrm{CH}_{3}\right), 0.84\left(\mathrm{~d}, J=6.4 \mathrm{~Hz}, 3 \mathrm{H}, 21-\mathrm{CH}_{3}\right)$, 0.73 (s, 3H, 18- $\mathrm{CH}_{3}$ ); IR (KBr) v: 3427, 2930, 2867, 1729, 1662, 1534, $1276 \mathrm{~cm}^{-1}$; ESI-MS $m / z(\%): 861\left[(\mathrm{M}+\mathrm{H})^{+}\right.$, 100]. Anal. calcd for $\mathrm{C}_{41} \mathrm{H}_{54} \mathrm{Cl}_{2} \mathrm{~N}_{6} \mathrm{O}_{6} \mathrm{~S}_{2}: \mathrm{C} 57.13, \mathrm{H} 6.31, \mathrm{~N}$ 9.75; found C 57.25, H 6.30, N 9.77.

$3 \alpha, 12 \alpha$-二 $\left[\left(N-1\right.\right.$ - 荟基- $N^{\prime}$-氨基甲酰氧基 $)$ 硫嫝-5 $\beta-24-$ 胆烷酸甲酯 $(\mathbf{5 j})$ : 黄色晶体，收率 $86 \%$. m.p. $121 \sim 122$ 
${ }^{\circ} \mathrm{C} ;[\alpha]_{\mathrm{D}}^{20}-45.45\left(c\right.$ 0.10, $\left.\mathrm{CH}_{2} \mathrm{Cl}_{2}\right) ;{ }^{1} \mathrm{H}$ NMR $\left(\mathrm{CDCl}_{3}, 400\right.$ MHz) $\delta: 10.54$ (s, 1H, NH), 10.22 (s, 1H, NH), 8.49 (s, 1H, $\mathrm{NH}), 8.28(\mathrm{~s}, 1 \mathrm{H}, \mathrm{NH}), 8.04(\mathrm{~s}, 1 \mathrm{H}, \mathrm{NH}), 7.81(\mathrm{~s}, 1 \mathrm{H}, \mathrm{NH})$, $7.91 \sim 7.38$ (m, 14H, Naphthyl-H), 5.04 (brs, $1 \mathrm{H}, 12 \beta-\mathrm{H}$ ), 4.69 (brs, $1 \mathrm{H}, 3 \beta-\mathrm{H}), 3.65\left(\mathrm{~s}, 3 \mathrm{H}, \mathrm{COOCH}_{3}\right), 0.94(\mathrm{~s}, 3 \mathrm{H}$, $\left.19-\mathrm{CH}_{3}\right), 0.79$ (d, $\left.J=6.4 \mathrm{~Hz}, 3 \mathrm{H}, 21-\mathrm{CH}_{3}\right), 0.71$ (s, 3H, $\left.18-\mathrm{CH}_{3}\right)$; IR ( $\left.\mathrm{KBr}\right) v: 3423,2964,2867,1726,1525,1450$, $1277 \mathrm{~cm}^{-1}$; ESI-MS $m / z(\%): 893\left[(\mathrm{M}+\mathrm{H})^{+}, 100\right]$. Anal. calcd for $\mathrm{C}_{49} \mathrm{H}_{60} \mathrm{~N}_{6} \mathrm{O}_{6} \mathrm{~S}_{2}: \mathrm{C} 65.89, \mathrm{H} 6.77, \mathrm{~N} 9.41$; found $\mathrm{C}$ 65.78, H 6.78, N 9.43.

\section{3 分子钳 $5 a \sim 5 j$ 的常规合成通法}

在 $50 \mathrm{~mL}$ 三颈瓶中加入 $0.2 \mathrm{~g}(0.5 \mathrm{mmol})$ 脱氧胆酸甲 酯中间体 3, $20 \mathrm{~mL}$ 无水 $\mathrm{CH}_{2} \mathrm{Cl}_{2}, 0.2 \mathrm{~mL}$ 无水吡啶, $0.11 \mathrm{~g}$ $(0.37 \mathrm{mmol})$ 三光气, 装上配有干燥管的冷凝管, 回流反 应 $6 \mathrm{~h}$, 中间体无须分离, 待反应物冷却至室温, 直接加 入 $1.2 \mathrm{mmol}$ 中间体 1 和 $0.2 \mathrm{~mL}$ 无水吡啶, 继续回流反 应 24 30 h (TLC 监测反应进程). 反应完成后, 减压蒸 去 $\mathrm{CH}_{2} \mathrm{Cl}_{2}$, 残余物加入 $20 \mathrm{~mL}$ 乙酸乙酯溶解, 过滤, 滤 渣用 $10 \mathrm{~mL}$ 乙酸乙酯洗涤，合并滤液，依次用 $5 \% \mathrm{Na}$ $\mathrm{HCO}_{3}(15 \mathrm{~mL} \times 3)$, 饱和食盐水 $(15 \mathrm{~mL} \times 3)$ 洗涤, 无水 $\mathrm{Na}_{2} \mathrm{SO}_{4}$ 干燥, 过滤, 减压蒸去溶剂, 柱层析分离纯化[固 定相用硅胶 $\mathrm{H}$, 洗脱剂为 $V$ (石油醚) $: V\left(\mathrm{CH}_{3} \mathrm{COO}-\mathrm{C}_{2} \mathrm{H}_{5}\right)$ $=8: 1 \sim 1: 1]$ 得纯品.

\section{4 识别性能测试}

以氯仿作溶剂, 固定主体分子钳的浓度在 $1 \times$ $10^{-5} \sim 10 \times 10^{-5} \mathrm{~mol} \cdot \mathrm{L}^{-1}$ 之间(由主体摩尔消光系数的大 小决定所配溶液浓度的大小), 不断加入客体分子, 其 浓度在 $10^{-4} \sim 10^{-3} \mathrm{~mol} \cdot \mathrm{L}^{-1}$ 间变化, 测定各组配合物溶 液的吸光度值, 为消除客体化合物在紫外区的干扰, 采 用相同浓度的客体氯仿液作参比, 同时为排除体积变化 对吸光度的影响, 主体中加入客体化合物的总体积不超 过 $100 \mu \mathrm{L}$. 测定温度控制在 $(25 \pm 0.1){ }^{\circ} \mathrm{C}$, 吸光度误差 不超过 0.002 .

\section{2 结果与讨论}

\section{1 微波法与常规法合成目标物 $5 a \sim 5 j$ 的对比}

两种不同加热方法合成目标物 $\mathbf{5 a} \sim \mathbf{5} \mathbf{j}$ 反应时间和 产率列于表 1 . 由表 1 可以看出, 微波法与传统加热合 成方法相比，具有以下优势: (1)反应速率增大了 84 91 倍, 大大缩短了反应时间; (2)目标分子钳的产率得到较 大的提高, 微波法的产率在 $81 \% \sim 86 \%$ 之间, 而传统加 热法的产率在 34\% 45\%之间, 这主要是因为微波反应 是从物质分子的内部进行加热, 具有极强的热效应, 可 以大大地降低反应的活化能, 使反应速度极大地提高.
由于微波反应的时间短，因此副反应少，副产物少，故 产率高; 而常规加热时间较长, 少则几个小时, 多则几 十个小时, 这样副反应多, 副产物较多, 所以产率较低. 因此在微波辐射条件下, 用三光气代替剧毒的光气，采 用一锅法, 是一种快速、有效、安全、绿色化地合成取 代氨基硫䐂型脱氧胆酸分子钳的好方法.

表 1 微波法与常规法合成目标物 $\mathbf{5 a} \sim \mathbf{5 j}$ 的比较

Table 1 Synthetic comparison of products $\mathbf{5 a} \sim \mathbf{5} \mathbf{j}$ between microwave irradiation and conventional heating

\begin{tabular}{|c|c|c|c|c|}
\hline Compd. & $\operatorname{Method}^{a}$ & $t / \min$ & Yield/\% & $t_{\mathrm{C}} / t_{\mathrm{MW}}{ }^{b}$ \\
\hline \multirow{2}{*}{$5 \mathbf{a}$} & $\mathrm{C}$ & 1800 & 36 & \multirow{2}{*}{90} \\
\hline & MW & 20 & 83 & \\
\hline \multirow{2}{*}{$5 b$} & $\mathrm{C}$ & 1980 & 34 & \multirow{2}{*}{86} \\
\hline & MW & 23 & 82 & \\
\hline \multirow{2}{*}{$5 c$} & $\mathrm{C}$ & 1920 & 42 & \multirow{2}{*}{91} \\
\hline & MW & 21 & 86 & \\
\hline \multirow{2}{*}{$5 d$} & $\mathrm{C}$ & 1920 & 45 & \multirow{2}{*}{91} \\
\hline & MW & 21 & 85 & \\
\hline \multirow{2}{*}{$5 e$} & $\mathrm{C}$ & 2160 & 40 & \multirow{2}{*}{86} \\
\hline & MW & 25 & 82 & \\
\hline \multirow{2}{*}{$5 f$} & $\mathrm{C}$ & 2040 & 43 & \multirow{2}{*}{85} \\
\hline & MW & 24 & 84 & \\
\hline \multirow{2}{*}{$5 \mathrm{~g}$} & $\mathrm{C}$ & 1860 & 44 & \multirow{2}{*}{84} \\
\hline & MW & 22 & 81 & \\
\hline \multirow{2}{*}{$5 \mathrm{~h}$} & $\mathrm{C}$ & 1800 & 45 & \multirow{2}{*}{86} \\
\hline & MW & 21 & 83 & \\
\hline \multirow{2}{*}{$5 \mathbf{i}$} & $\mathrm{C}$ & 1800 & 41 & \multirow{2}{*}{86} \\
\hline & MW & 21 & 84 & \\
\hline \multirow{2}{*}{$5 \mathbf{j}$} & $\mathrm{C}$ & 1800 & 39 & \multirow{2}{*}{90} \\
\hline & MW & 20 & 86 & \\
\hline
\end{tabular}

${ }^{a} \mathrm{C}$ : 传统加热方法, MW: 微波辐射法; ${ }^{b} t_{\mathrm{C}} / t_{\mathrm{MW}}$ : 两种加热方法所用时间之 比

\section{2 目标化合物 $5 \mathrm{a} \sim 5 \mathrm{j}$ 的结构确证}

在 ${ }^{1} \mathrm{H}$ NMR 谱图中, $\delta 10.50 \sim 7.60$ 为取代氨基硫艮 的 $\mathrm{NH}$ 的化学位移，而且其质子数相当于两个取代氨基 硫嫝结构单元连接在甾体上, $\delta 7.40 \sim 6.90$ 左右的多重 峰为取代氨基硫服结构中芳环的化学位移; 在 IR 谱图 中, 大约在 $3300 \mathrm{~cm}^{-1}$ 附近出现强的 $\mathrm{N}-\mathrm{H}$ 键的伸缩振 动吸收峰, 在 $1700 \mathrm{~cm}^{-1}$ 附近出现酰胺羰基 $(\mathrm{C}=\mathrm{O})$ 的伸 缩振动吸收峰; 从质谱图中可见, 化合物 5 均能给出分 子离子峰, 其 $m / z$ 与相应分子式的分子量是一致的. 元 素分析结果表明, 所有化合物的组成与其实验式相符.

综上所述，化合物 $\mathbf{5 a} \sim \mathbf{5 j}$ 所有光谱和元素分析数据 与其结构式符合, 表明所合成的化合物为目标化合物.

\section{3 主客体配合物的形成及其化学计量}

在紫外光谱滴定实验中，固定主体分子钳的浓度， 往主体溶液中加入不同浓度的客体阴离子溶液, 随着客 
体分子的不断加入，主体生色团的最大吸收呈规律性下 降, 表明客体分子进入主体分子裂穴并产生了识别配合 作用, 典型的吸收变化见图 1. 根据 Eq. 1, 在 $[\mathrm{G}]_{0} \gg$ $[\mathrm{H}]_{0}$ 时, 以 $1 /[\mathrm{G}]_{0}$ 对 $1 / \Delta A$ 作图, 均给出良好的线性关系, 以分子钳 $\mathbf{5 f}$ 为例, 结果如图 2 所示. 表明 $\mathrm{Cl}^{-}$与主体分 子钳产生了识别配合作用, 并形成 $1: 1$ 型超分子配合 物, 根据直线的截距和斜率计算出配合物的结合常数 $\left(K_{\mathrm{a}}\right)$, 再根据 Eq. 2 计算出自由能变化 $\left(-\Delta G^{0}\right)$. 主体分 子钳 $5 \mathrm{e}, \mathbf{5 f}, \mathbf{5 h}$ 对 $\mathrm{Cl}^{-}, \mathrm{Br}^{-}, \mathrm{I}^{-}, \mathrm{NO}_{3}^{-}$阴离子的结合常数 $\left(K_{\mathrm{a}}\right)$ 和自由能变化 $\left(-\Delta G^{0}\right)$ 列于表 2 .

$$
\begin{aligned}
\frac{1}{\Delta A} & =\frac{1}{a K_{\mathrm{a}}[\mathrm{G}]_{0}}+\frac{1}{a} \\
\Delta G^{0} & =-R T \operatorname{Ln} K_{\mathrm{a}}
\end{aligned}
$$

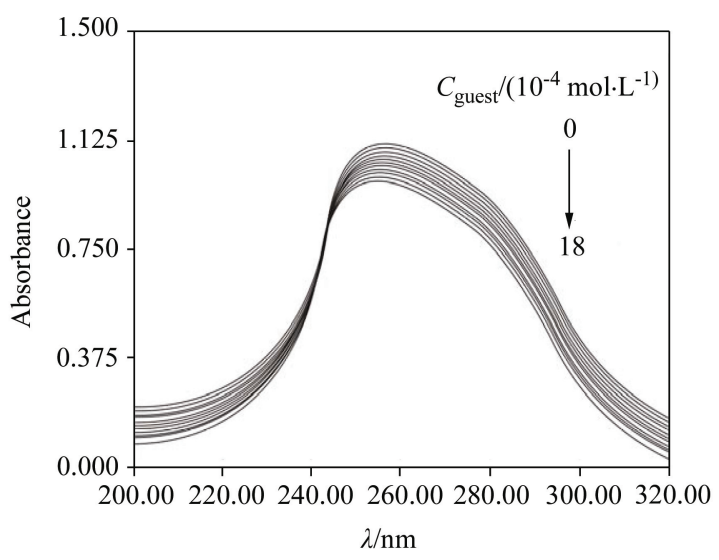

图 $15 \mathbf{f f}$ 中加入 $(n-\mathrm{Bu})_{4} \mathrm{~N}^{+} \mathrm{Cl}^{-}$的 UV 谱图

Figure 1 UV spectra of $\mathbf{5 f}$ in the presence of $(n-\mathrm{Bu})_{4} \mathrm{~N}^{+} \mathrm{Cl}^{-}$ $\lambda_{\max }=258 \mathrm{~nm} ; C_{5 \mathrm{f}}: 9.4 \times 10^{-5} \mathrm{~mol} \cdot \mathrm{L}^{-1} ; C\left(\mathrm{Cl}^{-}\right): 0,1.80 \times 10^{-4}, 3.60 \times$ $10^{-4}, 5.40 \times 10^{-4}, 7.20 \times 10^{-4}, 9.00 \times 10^{-4}, 10.80 \times 10^{-4}, 11.60 \times 10^{-4}$, $13.40 \times 10^{-4}, 15.20 \times 10^{-4}, 18.00 \times 10^{-4} \mathrm{~mol} \cdot \mathrm{L}^{-1}$

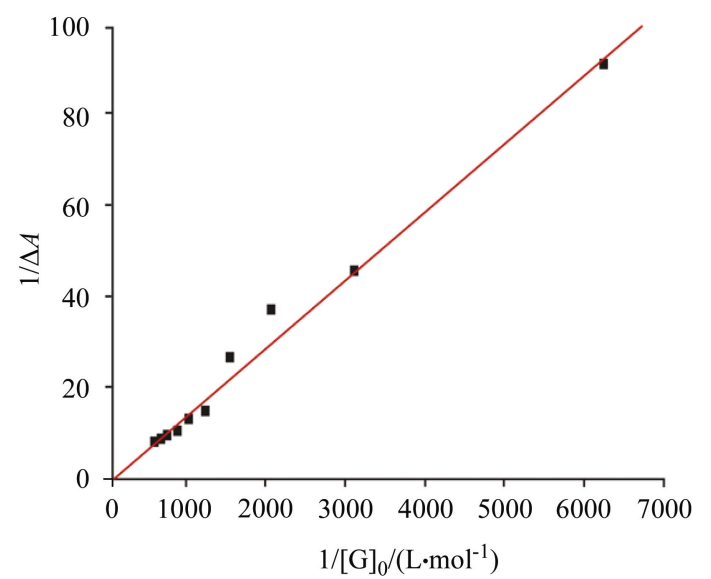

图 $225{ }^{\circ} \mathrm{C}$ 时 $\mathrm{CHCl}_{3}$ 中分子钳 $\mathbf{5 f}$ 与 $(n-\mathrm{Bu})_{4} \mathrm{~N}^{+} \mathrm{Cl}^{-}$形成配合物 的 $1 / \Delta A$ 对 $1 /[\mathrm{G}]_{0}$ 作图

Figure 2 Typical plot of $1 / \Delta A$ versus $1 /[\mathrm{G}]_{0}$ for the inclusion complex of molecular tweezer $\mathbf{5 f}$ with $(n-\mathrm{Bu})_{4} \mathrm{~N}^{+} \mathrm{Cl}^{-}$in $\mathrm{CHCl}_{3}$ at $25{ }^{\circ} \mathrm{C}$
表 $225{ }^{\circ} \mathrm{C}$ 时在 $\mathrm{CHCl}_{3}$ 溶液中分子钳 $\mathbf{5 e}, \mathbf{5 f}, \mathbf{5 h}$ 与阴离子形 成包结配合物的结合常数 $\left(K_{\mathrm{a}}\right)$ 和自由能变化 $\left(-\Delta G^{0}\right)$

Table 2 Association constants $\left(K_{\mathrm{a}}\right)$ and Gibbs free energy changes $\left(-\Delta G^{0}\right)$ for the inclusion complexes of anions with mo-

\begin{tabular}{|c|c|c|c|}
\hline Host & Guest & $K_{\mathrm{a}} /\left(\mathrm{L} \cdot \mathrm{mol}^{-1}\right)$ & $-\Delta G^{0} /\left(\mathrm{kJ} \cdot \mathrm{mol}^{-1}\right)$ \\
\hline \multirow{4}{*}{$5 e$} & $\mathrm{Cl}^{-}$ & 1112.00 & 17.37 \\
\hline & $\mathrm{Br}^{-}$ & 699.15 & 16.22 \\
\hline & $\mathrm{I}^{-}$ & 791.56 & 16.53 \\
\hline & $\mathrm{NO}_{3}^{-}$ & 304.31 & 14.17 \\
\hline \multirow{4}{*}{$5 f$} & $\mathrm{Cl}^{-}$ & 2720.69 & 19.58 \\
\hline & $\mathrm{Br}^{-}$ & 929.42 & 16.93 \\
\hline & $\mathrm{I}^{-}$ & 1106.97 & 17.36 \\
\hline & $\mathrm{NO}_{3}^{-}$ & 248.12 & 13.44 \\
\hline \multirow{4}{*}{$5 \mathrm{~h}$} & $\mathrm{Cl}^{-}$ & 1678.60 & 18.39 \\
\hline & $\mathrm{Br}^{-}$ & 279.62 & 13.95 \\
\hline & $\mathrm{I}^{-}$ & 542.47 & 15.60 \\
\hline & $\mathrm{NO}_{3}^{-}$ & 221.52 & 13.37 \\
\hline
\end{tabular}
lecular tweezers $\mathbf{5 e}, \mathbf{5 f}, \mathbf{5 h}$ in $\mathrm{CHCl}_{3}$ at $25{ }^{\circ} \mathrm{C}$

由表 2 可看出, 这类主体对所考察的阴离子具有良 好的识别能力, 主体与底物间形成了 $1: 1$ 型超分子配 合物, 所有主体对所考察的底物阴离子的识别结合常数 大小次序为: $\mathrm{Cl}^{-}>\mathrm{I}^{-}>\mathrm{Br}^{-}>\mathrm{NO}_{3}^{-}$, 其原因为 $\mathrm{NO}_{3}^{-}$是 三角型离子, 与主体匹配较差, 因此识别效果最差. $\mathrm{Cl}^{-}$, $\mathrm{I}^{-}, \mathrm{Br}^{-}$是球形离子, 能与主体很好的匹配, 此外 $\mathrm{Cl}^{-}$电 子云密度比 $\mathrm{I}^{-}, \mathrm{Br}^{-}$大，能与主体产生较强的氢键作用， 识别效果最好, 而 $\mathrm{I}^{-}$离子直径比 $\mathrm{Br}^{-}$大, 故与主体形成 氢键的能力较强(主体与客体形成氢键的强弱与距离有 密切的关系), 识别效果 $\mathrm{I}^{-}$优于 $\mathrm{Br}^{-}$, 所以对阴离子的识 别选择性显示为 $\mathrm{Cl}^{-}>\mathrm{I}^{-}>\mathrm{Br}^{-}>\mathrm{NO}_{3}^{-}$. 其次, 主体结构 的微小变化, 对结合常数存在明显的影响, 如: 主体 $\mathbf{5 e}$, 5f 手臂芳环上 $\mathrm{OCH}_{3}$ 基的位置分别位于芳环的邻位和对 位时, 其对 $\mathrm{Cl}^{-}$的结合常数分别为 $1112.00,2720.69$ $\mathrm{L} \cdot \mathrm{mol}^{-1}$. 这种由于主体手臂芳环取代基的位置变化所 造成的微环境效应对识别性能的影响, 不仅在理论上对 研究人工受体的设计合成和微环境效应具有重要意义, 而且可望在化学传感中具有潜在的应用前景.

\section{References}

[1] Martinez, M. R.; Sancenon, F. Chem. Rev. 2003, 103, 4419.

[2] Gong, W. T.; Bao, S.; Wang, F. R.; Ye, J. W.; Ning, G. L.; Hiratani, K. Tetrahedron Lett. 2011, 52, 630.

[3] Chahar, M.; Pandey, P. S. Tetrahedron 2008, 64, 6488.

[4] Kondo, S. I.; Nagamine, M.; Karasawa, S. Ishihara, M.; Unno, M.; Yano, Y. Tetrahedron 2011, 67, 943.

[5] Ghosh, K.; Kar, D.; Chowdhury, P. R. Tetrahedron Lett. 2011, 52, 5098 . 
[6] Chhatra, R. K.; Kumar, A.; Pandey, P. S. J. Org. Chem. 2011, 76, 9086.

[7] Xue, W. J.; Li, L.; Li, Q.; Wu, A. X. Talanta 2012, 88, 734.

[8] Zhao, Z. G.; Liu, X. L.; Li, Q. H.; Chen, S. H. Chin. J. Org. Chem. 2009, 29, 1336 (in Chinese).

(赵志刚, 刘兴利, 李清寒, 陈淑华, 有机化学, 2009, 29, 1336.)

[9] Lerouge, M. H.; Hudhomme, P.; Salle, M. Chem. Soc. Rev. 2011, 40,30 .

[10] Leblond, J.; Petitjean, A. ChemPhysChem 2011, 12, 1043.

[11] Polshettiwar, V.; Varma, R. S. Acc. Chem. Res. 2008, 41, 629.
[12] Kappe, C. O. Angew. Chem., Int. Ed. 2004, 43, 6250.

[13] Xiong, X. Q.; Cai, L.; Tang, Z. K. Chin. J. Org. Chem. 2012, 32, 1410 (in Chinese).

(熊兴泉, 蔡雷, 唐忠科, 有机化学, 2012, 32, 1410.)

[14] Qiu, L. Y.; Shi, Z. C.; Mei, Q. G.; Zhao, Z. G. J. Chem. Res. 2011, $35,456$.

[15] Zhao, Z. G.; Liu, X. L; Li, H.; Tang, X. L. Chin. J. Org. Chem. 2008, 28, 1233 (in Chinese).

(赵志刚, 刘兴利, 李晖, 唐晓丽, 有机化学, 2008, 28, 1233.)

(Zhao, C.) 